\title{
Author Correction: Chromatin IncRNA Platr10 controls stem cell pluripotency by coordinating an intrachromosomal regulatory network
}

Zhonghua Du ${ }^{1,2 \dagger}$, Xue Wen ${ }^{1+}$, Yichen Wang ${ }^{1,2+}$, Lin Jia ${ }^{1,2 \dagger}$, Shilin Zhang ${ }^{1,2}$, Yudi Liu ${ }^{1,2}$, Lei Zhou ${ }^{1,2}$, Hui Li ${ }^{1}$, Wang Yang ${ }^{1}$, Cong Wang ${ }^{1}$, Jingcheng Chen ${ }^{1}$, Yajing Hao ${ }^{3}$, Daniela Salgado Figueroa ${ }^{4}$, Huiling Chen ${ }^{2,5}$, Dan $\mathrm{Li}^{1}$,

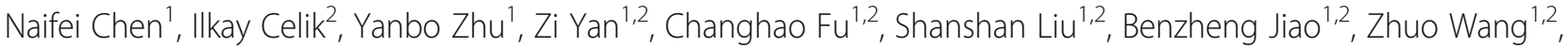
Hui Zhang ${ }^{6}$, Günhan Gülsoy ${ }^{7}$, Jianjun Luo ${ }^{3}$, Baoming Qin ${ }^{6}$, Sujun Gao ${ }^{1}$, Philipp Kapranov ${ }^{8}$, Miguel A. Estebann, Songling Zhang ${ }^{1}$, Wei Li ${ }^{1}$, Ferhat Ay ${ }^{4}$, Runsheng Chen ${ }^{3}$, Andrew R. Hoffman ${ }^{2^{*}}$, Jiuwei Cui ${ }^{1^{*}}$ and Ji-Fan Hu ${ }^{12^{*}}$

The original article can be found online at https://doi.org/10.1186/ s13059-021-02444-6.

* Correspondence: arhoffman@ stanford.edu; cuijw@jlu.edu.cn; jifan@stanford.edu; hujifan@jlu.edu. $\mathrm{cn}$

†'Zhonghua Du, Xue Wen, Yichen Wang and Lin Jia contributed equally to this work.

${ }^{2}$ Stanford University Medical School, VA Palo Alto Health Care System,

Palo Alto, CA 94304, USA

${ }^{1}$ Key Laboratory of Organ

Regeneration and Transplantation of Ministry of Education, Stem Cell and Cancer Center, First Hospital, Jilin University, Changchun, Jilin 130061, People's Republic of China Full list of author information is available at the end of the article

\section{Correction to: Genome Biol 22, 233 (2021)}

https://doi.org/10.1186/s13059-021-02444-6

Following publication of the original article [1], the authors identified an error in the author group. The author, Daniela Salgado Figueroa, had erroneously been omitted from the group. The author group has been updated in this correction article and the original article [1] has been corrected.

\begin{abstract}
Author details
'Key Laboratory of Organ Regeneration and Transplantation of Ministry of Education, Stem Cell and Cancer Center, First Hospital, Jilin University, Changchun, Jilin 130061, People's Republic of China. ${ }^{2}$ Stanford University Medical School, VA Palo Alto Health Care System, Palo Alto, CA 94304, USA. ${ }^{3}$ CAS Key Laboratory of RNA Biology, Institute of Biophysics, Chinese Academy of Sciences, Beijing 100101, People's Republic of China. ${ }^{4}$ La Jolla Institute for Allergy and Immunology, La Jolla, California 92037, USA. ${ }^{5}$ Department of Endocrinology, Xiangya Hospital, Central South University, Changsha, Hunan, People's Republic of China. ${ }^{6}$ Guangzhou Institutes of Biomedicine and Health, Chinese Academy of Sciences, Guangzhou, Guangdong 510530, People's Republic of China. ${ }^{7}$ Google Inc., Mountain View, CA 94043, USA. ${ }^{8}$ Institute of Genomics, School of Biomedical Sciences, Huaqiao University, Xiamen 361021, People's Republic of China.
\end{abstract}

Published online: 20 September 2021

\section{Reference}

1. Du Z, Wen X, Wang Y, et al. Chromatin IncRNA Platr10 controls stem cell pluripotency by coordinating an intrachromosomal regulatory network. Genome Biol. 2021;22(1):233. https://doi.org/10.1186/s13059-021-02444-6. 